\title{
Knowledge on breast cancer and practice of breast self examination among selected female university students in Malaysia.
}

\begin{abstract}
Breast cancer is the most common cancer affecting women in most parts of the world including Malaysia. Even though breast self examination (BSE) is not seen as a relevant cancer screening tool anymore, it still plays an important role in the breast health awareness programme. Aim of the study to determine the knowledge of respondents on breast cancer regarding the risk factors, symptoms, and to determine respondents' practice of breast selfexamination. A cross sectional study was carried out in University Putra Malaysia, data were collected using validated questionnaire developed for this study. Among respondents $197(83.1 \%)$ were single, 100 were Malay (42.3\%) and 49(20.7\%) of the respondents reported having a family history of breast cancer. eighty-seven respondents $(36.7 \%)$ claimed they had practice BSE. There were statistically significant differences between those who practice and did not practice BSE in term of knowledge regarding risk factors, symptoms of breast cancer, total knowledge of breast cancer and knowledge score of BSE (p-value <0.05). The findings showed that knowledge of breast cancer and the practice of BSE is inadequate among young Malaysian female.
\end{abstract}

Keyword: breast cancer; Breast awareness; Breast self examination; Malaysia. 